\title{
Extended High Efficiency Computed Tomography with Optimized Recursions
}

\author{
Wolfram R. Jarisch ${ }^{1}$, Michael J. Curry ${ }^{2}$, Peter J. Basser ${ }^{3}$
}

1. Cyber Technology, Inc., Potomac, MD, U.S.A.

2. CS Department, University of Maryland, College Park, U.S.A.

3. NICHD, National Institutes of Health, Bethesda, MD, U.S.A.

The objective of the novel extended High Efficiency Computed Tomography (CT) with Optimized Recursions (eHECTOR) is to improve the accuracy and rate of convergence of nonlinear iterative CT reconstruction when compared to traditional iterative reconstruction (IR) methods [1]. Traditional IR is initialized with linear Filtered Back-Projection (FBP) or Fast Fourier Transform (FFT) methods [2,3]. The absence of analytic approximations to perform subsequent iterations, however, requires iterative numerical methods (INM), such as SART, to refine and constrain the initial density estimate. In this way radiation exposure and measurement requirements can be reduced, when compared to pure FBP and FFT methods. The use of SART and related methods, however, uses a relaxation parameter that cannot produce optimal density relaxation steps and is computationally expensive [4].

By contrast, eHECTOR builds on concepts from HECTOR and HECT $[5,6]$. HECTOR estimates many pixel-associated relaxation factors; HECT, originally designed for angiographic reconstruction, introduces logarithmic weighting of pixel data. HECT, however, is limited by an empirical relaxation algorithm.

Improved convergence with eHECTOR follows from the use of many relaxation parameters, non-linear transformations, and analytic results applied to linearized density state updates within the non-linear estimation process. In particular, the highly contractive mapping (CM) of linear inversion methods is used effectively when operating on small signal residual errors. The effectiveness of CM is achieved through a combination of several ideas:

1. given monotone nonlinear projection data transformations and constraining voxel output data transformations, evaluate relaxation parameters from data transform inverse function slopes;

2. start the reconstruction with a single voxel representing the entire volume and single pixels representing individual projection densities;

3. iterate density estimates at any resolution until small signal update approximations are valid;

4. use a linear inversion method, such as the FBP or FFT, to process signal updates;

5. sub-divide densities into representations with higher resolution when small signal approximations are satisfied and sufficient convergence has been achieved;

6. update data values using estimates of the inverse slopes of non-linear transformations at the respective voxel and pixel densities as relaxation parameters for small signal gains;

7. terminate when, for example, projection residual errors are dominated by measurement noise and show minimal traces of the object of interest.

Several longstanding problems of tomographic reconstruction are solved with eHECTOR: (i) the traditional relaxation parameter is replaced with two large sets of parameters, typically in the order of $10^{8}$ elements. Each relaxation parameter is associated with an individual pixel or voxel density adjusting the small non-linear data transformation changes between iterations; (ii) gradual increase in resolution maintains operation near small signal approximations due to the effect of interpolation; (iii) the 
geometric nature of the expansion of the resolution of reconstructions makes the final full resolution reconstruction the dominant computing effort. The need of full resolution iteration may depend on factors such as the contrast properties of the object of interest.

Figures below compare reconstruction accuracy and speed of eHECTOR with SART [7]. Examples of eHECTOR reconstruction below used MATLAB code that slowed data I/O to the nVidia card. Using Python and several refinements of the present pipeline will bring further acceleration.

\section{References:}

[1] W Jarisch, U.S. Patent Application No. 15/816,745, Nov. 2017.

[2] B De Man, et al., 2005 IEEE Nuclear Science Symposium Conference Record.

[3] Y Zhang-O'Connor, J A Fessler, IEEE Trans. Medical Imag. 25 (2006).

[4] H Nien, J A Fessler, Rad. and Nuc. Med. (2015), p. 260.

[5] W Jarisch, Microscopy and Microan. Conf., Portland, OR, Aug., Late Breaking Poster LB15, 2010.

[6] W Jarisch, A C Dohnalkova, Microscopy and Microanalysis Conf., Richmond, VA, July, 2009.

[7] A Biguri et al, Biomedical Physics \& Engineering Express 2 (2016).

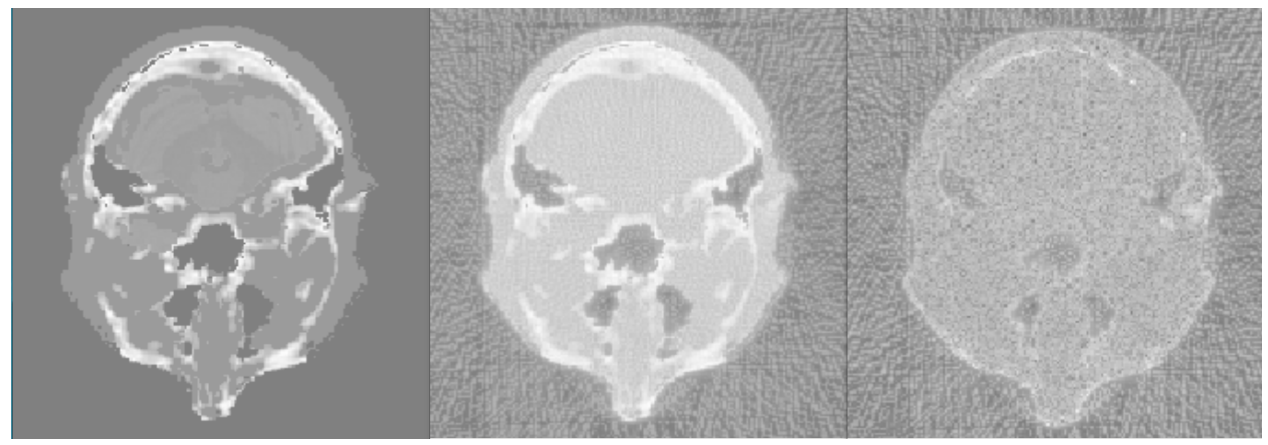

Figure 1. Slices through head phantom reconstructed with eHECTOR: left - slice though phantom; middle - slice through reconstruction; right - slice of residuals. Observe significantly reduced residuals compared to SART. Reconstruction time $\sim 50$ seconds at 200 projections in $256^{3}$ voxel volume on nVidia 1080. Note an order-of-magnitude speed-up compared to SART.

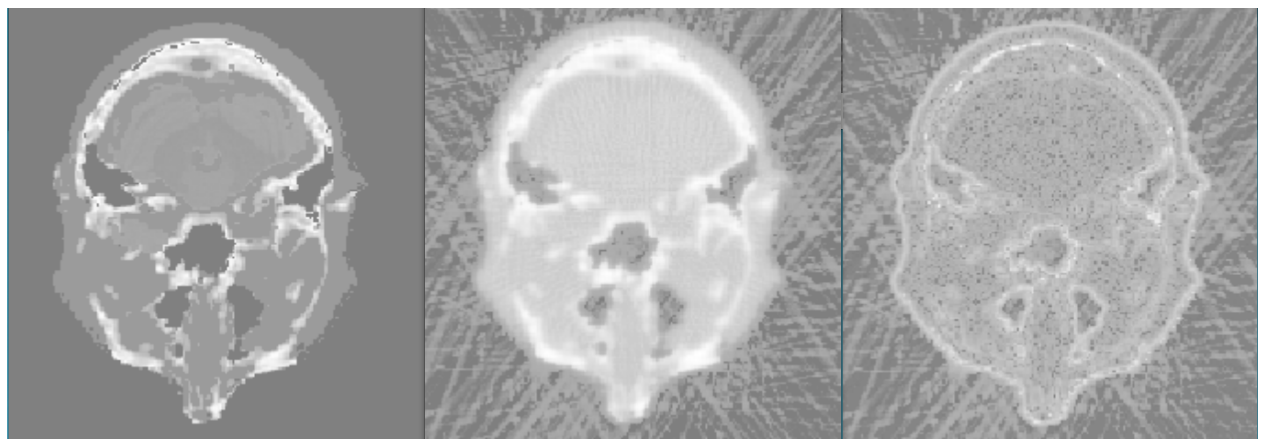

Figure 2. Slices through head phantom reconstructed with SART: left - slice though phantom; middle slice through reconstruction; right - slice of residuals. Observe blurred edges in SART reconstruction slice (middle). Phantom data and SART provided by [7]. Reconstruction time $\sim 10$ minutes at 200 projections in $256^{3}$ voxel volume on nVidia 1080. 